\title{
Design and Key Technology of Gardening Information Management System Based on Data Center
}

\author{
Xiguang Wang ${ }^{1}$, Weihua Lin ${ }^{1,2}$, Wen Zeng ${ }^{1,2}$ \\ ${ }^{1}$ Faculty of Information Engineering, China University of Geosciences, Wuhan, China \\ ${ }^{2}$ Wuhan Zondy Cyber T\&S. Co. Ltd, Wuhan, China \\ E-mail: wangxiguang@maggis.net
}

\begin{abstract}
To provide scientific management basis for the garden planning, project construction, maintenance, social service, this paper prompted that the urban gardening administration sectors need to construct gardening information management system. On the basis of fully requirements analysis of gardening sectors, this paper discussed the key technology for system construction. It also proposed to flexibly and smartly build up the system by using the secondary development design environment and running environment based on data center integration development platform. This system greatly helps the daily management and plays very important role in improving urban ecological environment and investment environment.
\end{abstract}

Keywords: Geographic Information System (GIS), Data Center Integrated Development Platform, Gardening, Workflow

\section{Introduction}

With economy rapidly development, gardening has been one of the most important parts of urban infrastructure construction which decorate the city more and more beautiful. More attention is paid to urban construction rather than urban management, which results in low informationized development. This problem has impacted the gardening construction and development. It mainly reflects in the following aspects:

1) Landscaping classification complexity result in difficult management, 2) due to the rapid development of urban construction, information is very difficult to update, 3) traditional management methods can't accurately statistic various landscaping, such as, old trees, parks, scenic spots, etc, 4) planning and evaluation are mainly based on practical experience.

Based on the above reasons, the key technology and construction methods of urban gardening need to be studied and gardening information management system will be studied. By operating this system, the operational level and management efficiency will be improved. Carry out comprehensive evaluation of urban gardening and set urban development strategies for gardening development.

\section{Objective of Urban Gardening Information Management System}

Urban gardening information management system is to study gardening classification encoding and management, data source sharing, data status, gardening 3D modeling, etc. All the landscapes, parks and scenic spots will be expressed in data and the urban basic spatial database, RS image data and gardening information base will be integrated. It will greatly help daily management, improve urban ecological environment, and play an important role in improving the investment environment.

1) Establish an integrated geographic information platform to achieve urban gardening and related data sharing, permanent preservation, real-time queries and dynamic management.

2) Establish administrative license handling platform. Integrate administrative licensing approval process and GIS to achieve digital approval process.

3) Establish decision-making support platform to help planning and designing, data query, simulated demolition, program optimization, etc.

4) Establish social service platform to release public urban gardening information. 


\section{System Structure Design}

Gardening information management system is based on MapGIS data center integrated development platform (data center for short) [1] to build and design on threelayer architecture, (shown in Figure 1). The first layer is hardware and software-based layer, which is base for management operation platform. Hardware part includes network equipment, servers, storage, backup devices, network including government special network, Internet, GPRS network, etc. Software part includes operating systems, database management systems, mirror and backup tools, GIS platforms and security software. The second layer is an integrated development platform for data center which is the running environment for gardening information system building and configuration. The third layer is the applications and services layer which provides business applications and service to users.

Integrated development platform for data center is based on the gardening information management requirements, which provides common needs and capabilities. It adopts service-oriented architecture conceptions. It designs and develops corresponding abstraction function module which constituted by several basic function composition. It can be divided into three layers, (shown in Figure 1). The first layer is to provide basic and common features, such as basic heterogeneous data view, GIS capabilities, remote sensing capabilities, 3D functions, data processing work space, data security rights management module, etc. The second layer is to provide basic and general landscape features, such as data models management, basic function management, gardening basic method management functions, etc. The third layer is to provide gardening professional functions, such as landscaping assisted analysis, removal and analysis, etc.

In addition, as for specific business needs, it provides a standard function module expansion interface, which supports particular business logic integration, specific business function development completion. It can also be incorporated into functional warehouse to be an integral part of a functional warehouse. In addition, data center integrated development module and function module adopts "Loosely coupled" connection approach. This approach which is flexible in structure and powerful expansibility is the best connection method which has minimal environmental impact. Operation adopts "service" approach which converts "data access operation" to "data access service request service". "Data access service" is an example, which fully embodies the latest "serviceoriented" design ideas.

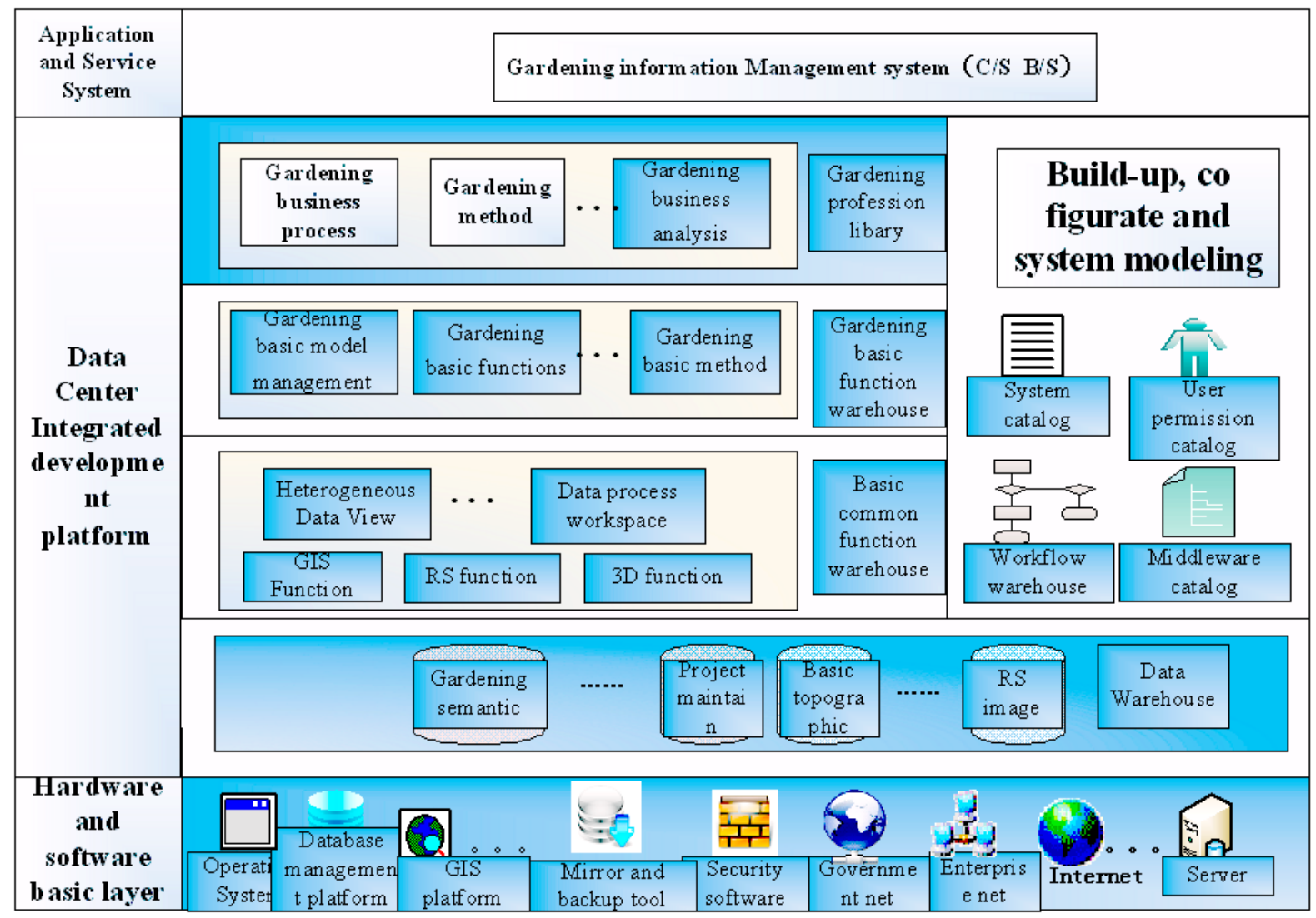

Figure 1. Gardening information management system layer structure. 


\section{System Function Design}

Urban gardening information management system mainly includes the following aspects:

\subsection{Establish Gardening Data Classification Dictionary}

Green classification corresponds to urban land can be divided into three layers, which has 29 categories, including parks green, production green, protection green land, subsidiary green land and other green space, trees, old trees, plant resource protection base, etc. According to urban greening classification standards, establishing data dictionary is the basis for gardening effective management, data sharing and dynamic updating. The classification coding should have the following characteristics, scientific, systematic, stable, integral, compatible, scalable, adaptable and flexible [2].

\subsection{Unified to Manage Various Data and Materials}

Gardening includes all kinds of thematic data. Business layer includes community green space, trees, old and valuable trees, etc. Engineering class includes green space system planning, detailed planning, project planning and design programs, urban gardening, long-term development plans and annual plans and other documents. In addition to basic spatial data and urban RS image information, there are lots of other spatial data, such as, water resources, meteorology, ecology, pests and diseases.

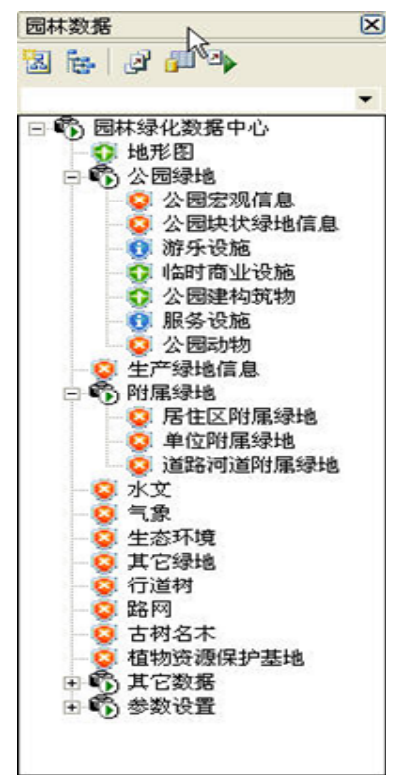

Figure 2. Gardening data center manager.
The data manager provided by data center can classify, store, manage, query, index and statistic the heterogeneous spatial data and non-spatial data by different region, different time era and different category.

\subsection{Gardening Plan Assisted Analysis}

By spatial overlay analysis of landscape planning and residents feature, the gardening green engineer involved in demolition and resident resettlement assignments will be obtained. Then according to the cost checking database attribute, the related fees can be got. Comprehensively analyze gardening planning of landscaping construction costs and adjust the green structure and layout in order to minimize risk and maximize efficiency.

\subsection{Establish Gardening 3D View}

By using 3D view model evaluation to display gardening design planning aesthetics. Dynamically generate 3D modeling by the combination of DEM, RS image, texture, planning graphs and digital topographic graph. User can observe different perspectives and gardening design effects which will help them to make appropriate adjustment.

\subsection{Dynamic Approval Process Update}

Through workflow, the administrative approval licensing process and GIS are closely integrated, which achieves seamless integration of GIS, MIS (Management Information System) and OA (Office Automation). According to approval licensing information, user can dynamically modify gardening information in related areas to ensure real time data [3].

\subsection{WEB Publishing Gardening Information and Public Services}

It provides information publishing and query of parks, green area, place of interest, etc. All the parks distribution and park activities are published on the internet. Citizen can plant and safeguard the greening by the published information.

\section{System Key Technology}

\subsection{Data Center}

Data center architecture is based on a new generation of GIS technology and new development model, which integrates "basic" and "application”.

Data center is both a "resource manager" and also a 
“system developer”. "Resource manager” manages data resource and function resource. Data resource is managed by data warehouse and function resource is managed by function warehouse [4,5]. "System developer" adopts flexible design concept which can build up the function very fast and adjust the function module according to the requirements. It has realized "zero-programming, smart combination and easy building-up” visual development.

\subsection{Landscaping Modeling}

Digital Elevation Model (DEM for short) is an entity ground model which uses an array of values to describe ground elevation. It's one of branches of Digital Terrain Model (DTM for short). The other digital model uses matrix form to express various non-terrain features, including the natural and geographical factors as well as the ground-related socio-economic and cultural factors, such as soil type, land use types, rock depth, land, commercial advantages district, etc. By using DEM, 3D visual reality technology, establish 3D view model of gardening planning area, such as urban green space system planning models, landscaping professional planning and green space system detailed planning model, landscape greening proportion and distribution models, urban green space and scenic spots of the planning model.

\subsection{Spatial Data Management}

Base state with amendments is one of spatial data management method which doesn't store all the status of researched area, but only the state of some point and also change to relative basic state. Base state with amendments can greatly reduce temporal data amount.

Gardening historical data management is based on tuple level which is based on base state with amendments. Generally, the status after construction of urban gardening is taken as "base state". User most concerned "current state" is the latest update data state. All the "current state” gardening information will be effectively managed which can fully reflect the change and development of gardening, and reduce historical data of the redundant and facilitate historical data management.

\subsection{Workflow Technology}

The workflow model based on network control realizes flexible operation adjustment and customization, which achieves seamless integration of GIS and office automation. The topological relations can realize automatically conditional judgment, loop, and countersigned, etc.

In this model, the "node" represents the workflow unit of the landscaping business departments. "Net line” represents the workflow between different departments, such as, the connection between Secretary's Office and Comprehensive Sectors, that is, the workflow transformation has converted to network resource flow. Workflow control also becomes a network control which makes the business logic of different sectors visualized, the logic control easier, faster and more accurate.

\section{System Building-up Process}

\subsection{Professional Base and Industry Professional Function Building}

Urban gardening information management system professional basic function includes, greening data model, metadata management, gardening basic function warehouse, gardening basic method warehouse, data exchange components, etc. (Shown in Figure 3). The basic function library includes data management basic function library, data update basic function library, data analysis basic function library, 3D model, encoding engine, etc.

System application function construction includes application function library, business process library, etc. The application function library includes analysis function library, business function library, thematic map

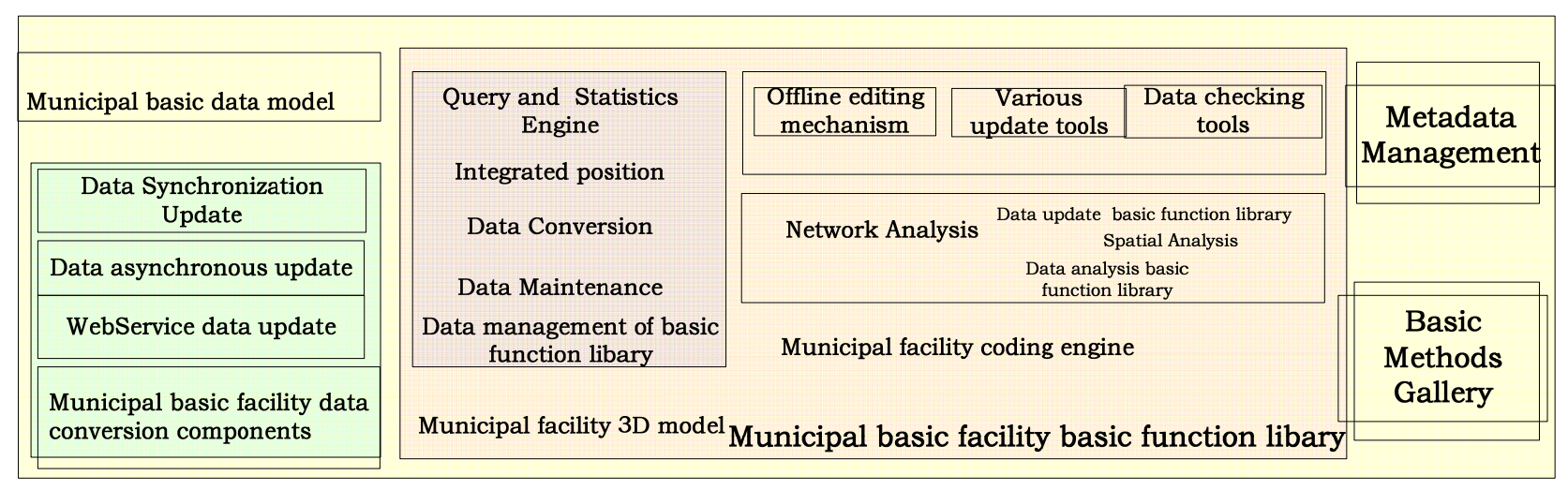

Fiugre 3. Professional basic function components. 
function, etc. Analysis function libraries includes landscaping demolition cost accounting, green analysis, greening comparative analysis, index analysis, etc. Gardening business functions includes gardening project management, landscape planning and management, garden maintenance, etc.

Both professional basic and industry function support Visual Studio2005. All the plug-ins development is according to the relative interface standards. After completing plug-in functions, find appropriate registration documents (*. rgs) and start editing plug-ins register in accordance with registration standards.

\subsection{Application System Building}

\subsubsection{System Building Process}

Building and configuration development model uses flexible design concept, which provides fast building-up and adjustment according to requirements. First all the configuration tools should in accordance with gardening business requirements, such as, data center designer, workflow designer and the user permissions designer, then design the system and form system solution in XML file format. When the system is running, the solution will be loaded in the scalable framework by the running environment, and thus to building up the gardening information management system, as shown in Figure 4.

\subsubsection{System Function Building}

Using integrated designer to complete system interface design (such as, system's right-click menu, system menu, tool bar, status bar, hot key, interactivity, etc) and hierarchical data catalog configuration. Workflow designer supports flexible administrative examination and approval process customization. User permission configuration tools can define system user, user role, user permission, user department, region, facility type. Add related permission menu and toolbar according to role, thus to provide permission distribution for user building system and realize combination of permission and business. Finally building up system main interface through data center, as shown in Figure 5.

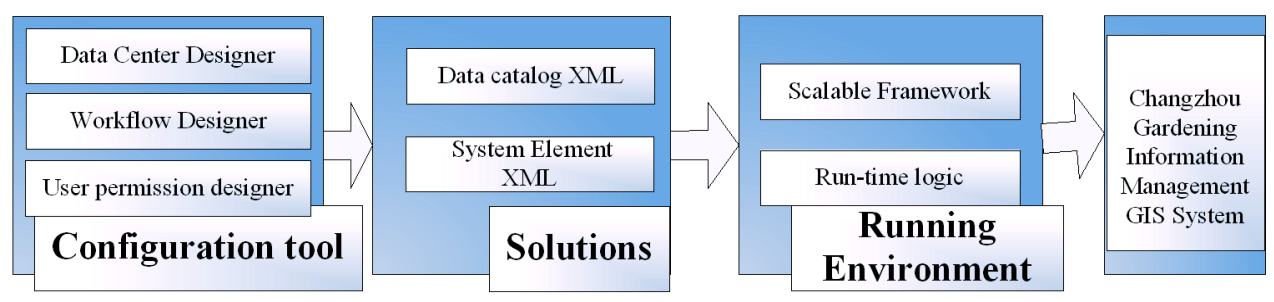

Figure 4. Urban gardening information system construction process.

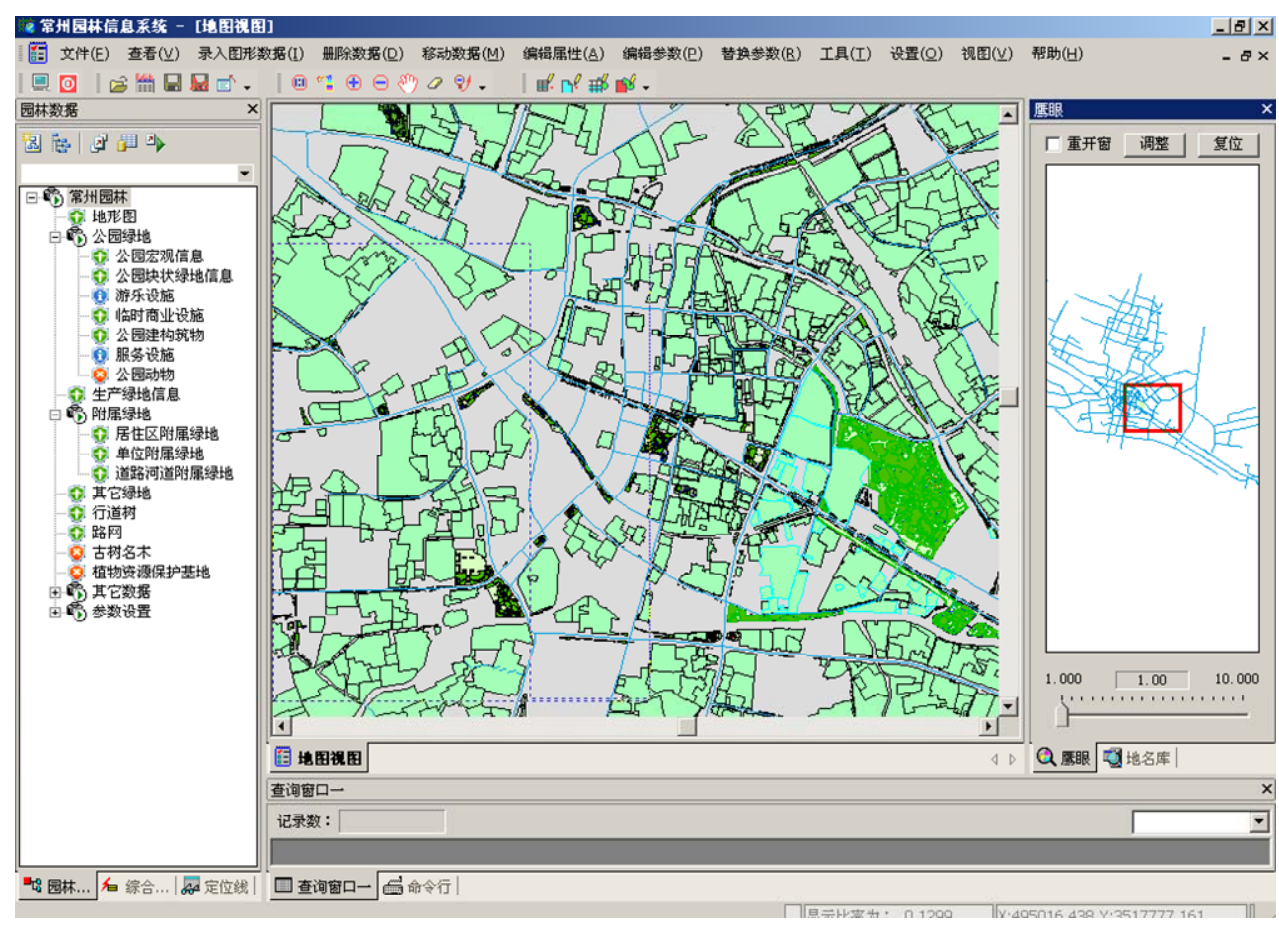

Figure 5. Gardening information management system main interface. 


\section{Conclusions}

Gardening information management system integrates urban basic spatial databases, gardening professional database and non-spatial database which help to fully grasp urban gardening status, and monitor and evaluate urban greening information. By integration of administrative examination and approval process and GIS, and in support of network technology, the gardening unit can carry out administrative and operational work in unified workflow framework. It improves information management level and realizes “digital gardening”.

\section{References}

[1] X. C. Wu, “Theory Technique and Application of GIS,”
China University of Geosciences Press, Wuhan, 2000.

[2] Y. Zheng, J. T. Jiang, J. B. He, et al., "Manual of Standardization of Urban Geographic Information Systems," Science Press, Beijing, 1998, pp. 20-21.

[3] W. Zeng and D. J. Zhang, "Scheme and Key Techniques for GIS-Based Integration of Municipal Management Information," Earth Science-China University of Geosciences, Vol. 31, No. 5, 2006, pp. 688-692.

[4] X. C. Wu and L. Wu, "Service-Oriented Distributed Spatial Information Supporting System," Earth ScienceChina University of Geosciences, Vol. 31, No. 5, 2006, pp. 585-589.

[5] X. C. Wu, "Datacenter Integration Development Technology: The Next Generation GIS Architecture and Development Model," Earth Science-China University of Geosciences, Vol. 34, No. 3, 2006, pp. 1-7. 